\title{
Spin and Statistics of Solitons and $J$-Homomorphism
}

\author{
Douglas C. Ravenel ${ }^{1}$ and A. Zee ${ }^{2}$ \\ 1 Department of Mathematics, University of Washington, Seattle, WA 98195, USA \\ 2 Department of Physics, University of Washington, Seattle, WA 98195, USA
}

\begin{abstract}
The deep connection between the spin and statistics of solitons and homotopy groups is clarified by using $J$-Homomorphism.
\end{abstract}

The deep connection between the spin and statistics of solitons and homotopy groups provides a fascinating link between physics and mathematics [1,2]. Recently, this connection has been studied in the context of concrete field theoretic models $[3,4]$ with possible relevance to the real world. The purpose of this paper is to sharpen the existing analysis and to bring the notion of $J$-homomorphism to the attention of the physics community.

Consider a generalized "nonlinear $\sigma$-model" in which the field $\varphi$ (or the order parameter in condensed matter physics) is restricted to lie on a manifold $M$. If the homotopy $\pi_{n}(M)$ is nontrivial, then topologically stable solitons exist in $n$-dimensional space, as is well-known. (Space does not mean space-time here.) A soliton is specified by a function $\varphi: R^{n} \rightarrow M$. The energy functional has the generic and schematic form with $\varphi(\mathbf{x}) \in M$, where $R^{n}$ is Euclidean space.

$$
E=\int d^{n} \mathbf{x}\left(\frac{\partial \varphi}{\partial \mathbf{x}}\right)^{2}
$$

It is understood that the indicated operations, differentiation and scalar product, can be defined. (The most common examples studied by physicists have $M=S^{n}$, taken to be the subset $\left\{\varphi: \varphi^{2}=1\right\}$ of $R^{n+1}$, on which $S O(n+1)$ acts by matrix multiplication.) In order that the soliton have finite energy one requires $\frac{\partial \varphi}{\partial \mathbf{x}}$ to vanish rapidly at $\infty$, i.e. $\varphi(\mathbf{x}=\infty)=\varphi_{0}$, a constant. Since $\varphi(\mathbf{x})$ is effectively constant outside a compact region, we may consider $\varphi$ to be defined on $B^{n}$, the unit ball. For example, the soliton corresponds to the map $B^{n} \stackrel{p}{\longrightarrow} S^{n} \stackrel{f}{\longrightarrow} M$. Here $f$ is a representative map of an element of $\pi_{n}(M)$ and the pinch $p$ is defined by

$$
p(\mathbf{x})=\left\{\begin{array}{lll}
(\mathbf{x} \sin \pi|\mathbf{x}| /|\mathbf{x}|, \cos \pi|\mathbf{x}|) & \text { if } \quad \mathbf{x} \neq 0 \\
(0,1) & \text { if } \quad \mathbf{x}=0
\end{array}\right.
$$


The boundary of $B^{n}=\partial B^{n}=S^{n-1}$ is mapped into a single point $(0,-1)$, the "south pole," on $S^{n}$. The map $f p$, suitably interpreted, is known as a Skyrmion in the physics literature [5].

To discuss the spin and statistics of the soliton, one has to introduce the notion of time. We go back to the basic notion of angular momentum and rotate the soliton through $2 \pi$ over a long time period $T$. In quantum mechanics, the state acquires a phase factor $e^{i 2 \pi J}$ at the end of this rotation, where $J$ is the angular momentum of the solution. This rotation acts on the field $\varphi$ and defines a family of functions $R \times B^{n} \rightarrow M$ by the formula $\tilde{\varphi}(t, \mathbf{x})=\varphi\left(R_{t} \mathbf{x}\right)$. When $R_{t}$ is the rotation at time $t$; this family satisfies $\tilde{\varphi}(0, \mathbf{x})=\varphi(\mathbf{x})=\tilde{\varphi}(T, \mathbf{x})$ and $\tilde{\varphi}(t, \mathbf{x}=\infty)=\varphi_{0}$; by writing $\tilde{\varphi}(t, \mathbf{x})=f_{t} p$ we get a family $\left\{f_{t}\right\}_{0 \leqq t \leqq T}$ of maps, $S^{n} \rightarrow M$ which, because of the periodicity over time $T$, defines a map $S^{1} \times S^{n} \rightarrow M$. We denote the homotopic class of these maps by $\left[S^{1} \times S^{n}, M\right]^{\prime}$, where the prime reminds us of the special condition $\tilde{\varphi}(t, \mathbf{x}=\infty)=\varphi_{0}$. To determine the statistics, we exchange a pair of solitons.

How is the preceding related to homotopy groups? To see the connection we have to go to an alternative point of view in determining spin and statistics. One thinks of the path integral formulation of quantum mechanics and considers the following history. Start at $t=-\infty$ with the vacuum state, i.e. a configuration $\varphi(t=-\infty, \mathbf{x})=\varphi_{0}$ for all $\mathbf{x}$. As time evolves, one causes a pair of soliton and antisoliton to be created and to be separated to a large distance from each other. One rotates the soliton through $2 \pi$ and then brings the soliton to the anti-soliton and allows them to annihilate, thus ending up at $t=+\infty$ again in the vacuum configuration. Since the function $\tilde{\varphi}(t, \mathbf{x})$ corresponding to this history goes to the constant $\varphi_{0}$ at the boundary of spacetime, it clearly defines a map of $S^{n+1} \rightarrow M$. The basic principles of quantum mechanics tell us that the phase $e^{i 2 \pi J}$ acquired by the wave function describes the angular momentum $J$ of the soliton. Now, if $\pi_{n+1}(M)=Z_{2}$, then the phase can only be +1 or $-1=e^{i \pi}$. Thus, one can have either $J$ integral or $J$ half-integral. On the other hand, if $\pi_{n+1}(M)=Z$, the phase is not constrained by homotopic considerations and may in general take on an arbitrary value $e^{i \theta}$. The physics with spin neither integral or half-integral and obeying statistics neither Bose-Einstein or Fermi-Dirac has been developed in a series of papers $[6,4,7]$. To determine the statistics, one considers a different history: two soliton-anti-soliton pairs are created and separated, then the two solitons are interchanged, and finally the solitons and the anti-solitons are allowed to annihilate. The function $\tilde{\varphi}(t, \mathbf{x})$ again corresponds to an element of $\pi_{n+1}(M)$.

The relation between the processes described in the two preceding paragraphs is best explained by referring to Fig. 1 . We have pictured the process of creating and annihilating a soliton and anti-soliton pair. The smaller rectangle frames a "close-up" shot of the $2 \pi$ rotation of the soliton. By "putting" an element of $\left[S^{1}\right.$ $\left.\times S^{n}, M\right]^{\prime}$ into the smaller rectangle, one obtains an element of $\pi_{n+1}(M)$ described by the larger rectangle.

We had studied [4] in detail the case $M=S^{2}$. Since $\pi_{3}\left(S^{2}\right)=Z$, we have a concrete example of solitons with peculiar spin and statistics. In contrast, in the case studied by Witten [3], $M=S^{3}$, and the solitons are required by the homotopy $\pi_{4}\left(S^{3}\right)$ to be either bosons or fermions. The fascinating possibility that the observed baryon may correspond to a soliton of this type has been suggested $[3,8$, 9]. 


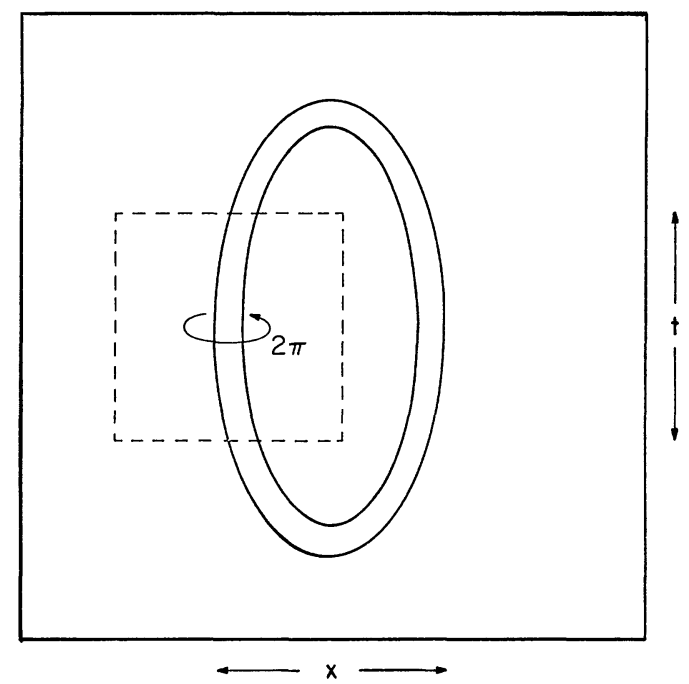

Fig. 1. Determination of the angular momentum of the soliton; see text for detailed explanation

It is worth noting that as a special case of the Freudenthal suspension $\Sigma$ : $\pi_{n+k}\left(S^{n}\right) \rightarrow \pi_{n+k+1}\left(S^{n+1}\right)$ these two discussions are related by $\pi_{2}\left(S^{2}\right) \rightarrow \pi_{3}\left(S^{3}\right)$, $\pi_{3}\left(S^{2}\right) \rightarrow \pi_{4}\left(S^{3}\right)$. (For the explicit maps, see Ref. 4.) Freudenthal proved that the suspension is onto for $n=k+1$ and an isomorphism for $n>k+1$.

We recall that in standard treatment of [10] angular momentum and quantum statistics one considers the first homotopy of the rotation group $\pi_{1}(S O(d))$. For $d \geqq 3$, it is known that [11] $\pi_{1}(S O(d))=Z_{2}$; thus, the rotation group admits double-valued representations and angular momentum can be half-integral. In contrast, $\pi_{1}(S O(2))=Z$. The angular momentum algebra is abelian in 2-dimensional space and so angular momentum is not quantized.

We ended Ref. 4 by remarking that there ought to be a connection between the first homotopy of the rotation groups and the homotopy of spheres, since $\pi_{1}(S O(2)) \sim \pi_{3}\left(S^{2}\right)$ and $\pi_{1}(S O(3)) \sim \pi_{4}\left(S^{3}\right)$. Indeed, there is. As we will see, the connection can be understood in rather physical terms.

We now describe the $J$-homomorphism [12] which maps $\pi_{k}(S O(n)) \rightarrow \pi_{n+k}\left(S^{n}\right)$. Given a representative of an element of $\pi_{k}(S O(n))$, i.e. a map $R: S^{k} \rightarrow S O(n)$, we are to construct a map $J(R): S^{n+k} \rightarrow S^{n}$. We first define the map $\tilde{R}: S^{k} \times B^{n} \rightarrow B^{n}$ by sending $(\mathbf{t}, \mathbf{x})$ to $R(\mathbf{t}) \mathbf{x}$ where $\mathbf{t}$ with $|\mathbf{t}|=1$ is in $S^{k}$ and $\mathbf{x}$ with $|\mathbf{x}| \leqq 1$ is in $B^{n}$. Now we can pinch $B^{n}$ to $S^{n}$ by the map $p$ described earlier. Next, we pinch $S^{k} \times B^{n}$ to $S^{n+k}$ by an onto map $s$ defined by $s(\mathbf{t}, \mathbf{x})=\left(\mathbf{t} \sqrt{1-\mathbf{x}^{2}}, \mathbf{x}\right)$. The restriction of $s$ to the boundary $\partial\left(S^{k} \times B^{n}\right)=S^{k} \times S^{n-1}$ is just projection onto $S^{n-1} C S^{n+k}$. (Note that this pinching is not to be confused with another pinching defined by identity $\left(S^{k}\right) \times p$. Recall that $S^{1} \times B^{1}$ can be pinched to either $S^{2}$ or the torus.) It can be shown [12] that the homotopy class of $J(R)$ depends only on that of $R$.

The map $s$ is invertible except on $S^{n-1}$. Explicitly, $s^{-1}(\mathbf{v}, \mathbf{w})=\left(\mathbf{v} / \sqrt{1-\mathbf{w}^{2}}, \mathbf{w}\right)$ with $v^{2}+w^{2}=1$. We define $J(R)$ to be $p \widetilde{R s^{-1}}$. On $S^{n-1}$ we let the image of $J(R)$ be 
$(0,-1)$. In summary, the $J$-homomorphism is constructed as follows

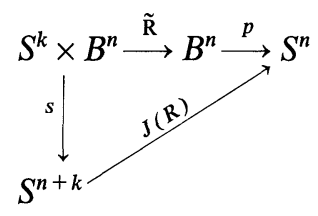

$J$ is an isomorphism for $k=1$ but not in general.

The above is a precise construction of $J$. Most physicists, however, would be content with an explicit formula for $J(R)$. Let $S^{n+k}=\left\{(\mathbf{t}, \mathbf{x}), \mathbf{t} \in R^{k+1}, \mathbf{x} \in R^{n}\right.$, with $\left.x^{2}+t^{2}=2\right\}$. Then, given $R$, we define

$$
J(R)(\mathbf{t}, \mathbf{x})=\left\{\begin{array}{lll}
\left(|\mathbf{t}| R(\mathbf{t} /|\mathbf{t}|) \mathbf{x}, 1-\mathrm{t}^{2}\right) & \text { if } \quad t \neq 0, \\
(0,1) & \text { if } \quad t=0 .
\end{array}\right.
$$

All of this is highly mathematical. But, in fact, it is casy to relate heuristically all this to the physical process of rotating the soliton described earlier. Let the rotation $R(\mathbf{t})$ be parametrized by $\mathbf{t} \in S^{k}$. (In the actual physical process we take $k=1$.) The set of rotations through $2 \pi$ describes an element of $\pi_{k}(S O(n))$ while the soliton $\varphi(\mathbf{x})$ describes an element of $\pi_{n}(M)$. Thus, the function $\varphi(R(\mathbf{t}) \mathbf{x})$ defines a map of $S^{k} \times S^{n} \rightarrow M$. Therefore, physics naturally leads us to a map of $\pi_{k}(S O(n)) \times \pi_{n}(M) \rightarrow\left[S^{k} \times S^{n}, M\right]^{\prime}$. We want a map into $\pi_{k+n}(M)$ rather than $\left[S^{k} \times S^{n}, M\right]^{\prime}$. (See earlier.) The $J$-homomorphism allows us to define precisely the desired map:

$$
\pi_{k}(S O(n)) \times \pi_{n}(M) \stackrel{J}{\longrightarrow} \pi_{n+k}\left(S^{n}\right) \times \pi_{n}(M) \stackrel{c}{\longrightarrow} \pi_{n+k}(M) .
$$

The composition $c$ defines a map $S^{n+k} \rightarrow M$ by multiplying $S^{n+k} \rightarrow S^{n}$ with $S^{n} \rightarrow M$.

We end with a few remarks about homotopy.

(1) Since $\pi_{1}(S O(n))=Z_{2}$ for $n>2$ one might think that if $\pi_{n}(M) \neq 0$ then $\pi_{n+1}(M)$ must be either 0 or $Z_{2}$. This is not true. A counter-example is provided by $\pi_{8}\left(S^{3}\right)=Z_{2}$ and $\pi_{9}\left(S^{3}\right)=Z_{3}$. We believe that in this case the corresponding soliton must be quantized as a boson.

(2) We can use the composition $c$ to write, for example,

$$
\pi_{4}\left(S^{3}\right) \times \pi_{3}\left(S^{2}\right) \rightarrow \pi_{4}\left(S^{2}\right), \quad \pi_{7}\left(S^{4}\right) \times \pi_{4}\left(S^{3}\right) \rightarrow \pi_{7}\left(S^{3}\right)
$$

and so on. Thus, the Hopf map $S^{3} \rightarrow S^{2}$ and its suspension allows us to conclude that an $S O(3)$ nonlinear $\sigma$-model in 3-dimensional space may be quantized as a fermion $\left(\pi_{4}\left(S^{2}\right)=Z_{2}\right)$.

(3) Various properties of $J$ are known to mathematicians, of course. For instance, the diagram

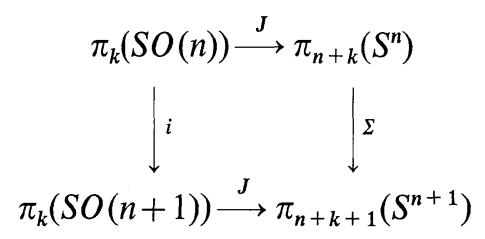

commutes where $i$ is induced by the inclusion of $S O(n)$ into $S O(n+1)$. 
Like $\Sigma, i$ is an isomorphism for $n>k+1$ and onto for $n=k+1$. This diagram provides a connection between various discussions in the physics literature $[3,4]$.

(4) $J$ allows us to generate various maps. For example, $\pi_{3}(S O(3)) \stackrel{J}{\longrightarrow} \pi_{6}\left(S^{3}\right)$. Since $S U(2) \sim S^{3}$, the double covering of $S O(3)$ by $S U(2)$ generates $\pi_{3}(S O(3))$.

Acknowledgements. One of us (A.Z.) thanks F. Wilczek for helpful discussions and V.P. Nair for a careful reading of the manuscript and for calling our attention to the paper of Williams. This research was supported in part by the National Science Foundation under Grant No. PHY 77-27084, supplemented by funds from the National Aeronautics and Space Administration.

\section{References}

1. Finkelstein, D., Rubinstein, J.: J. Math. Phys. 9, 1762 (1968)

2. Mickelsson, F.: Helsinki preprint

3. Williams, J.G.: Math. Phys. 11, 2611 (1970)

Witten, E.: Nucl. Phys. B 223, 422, 433 (1983)

4. Wilczek, F., Zee, A.: Phys. Rev. Lett. 51, 2250 (1983)

5. Skyrme, T.H.R.: A non-linear theory of strong interactions. Proc. Roy. Soc. London 247 A, 260 (1958); A non-linear field theory. 260 A, 127, (1961); Nucl. Phys. 31, 556 (1962)

6. Wilczek, F.: Phys. Rev. Lett. 48, 1144 (1982); 49, 957 (1982)

7. Wilczek, F., Zee, A.: UCSB ITP preprint NSF-ITP-83-148

8. Balachandran, A.P., Nair, V.P., Rajeev, S.G., Stern, A.: Phys. Rev. Lett. 49, 1124 (1982); Phys. Rev. D. 27, 1153 (1983)

9. Adkins, G., Nappi, C., Witten, E.: Princeton preprint (1983)

10. Wigner, E.P.: Group theory and its applications to the quantum mechanics of atomic spectra. New York: Academic Press 1959

11. Encyclopaedic Dictionary of Mathematics, for instance

12. Whitehead, G.W.: Elements of homotopy theory, p. 504. Berlin, Heidelberg, New York: Springer 1978

Communicated by A. Jaffe

Received May 18, 1984; in revised form October 15, 1984 
